\title{
A New Ultracompact Narrow Bandpass Microstrip Filter Using Double-Negative Quasiplanar Cells
}

\author{
Mohammad Reza Khawary, ${ }^{1}$ Vahid Nayyeri $\mathbb{D D}^{1,2}$ Seyed Mohammad Hashemi ${ }^{10}{ }^{3}$ \\ and Mohammad Soleimani ${ }^{1}$ \\ ${ }^{1}$ Antenna and Microwave Research Laboratory, Iran University of Science and Technology, Tehran, Iran \\ ${ }^{2}$ School of Advanced Technologies, Iran University of Science and Technology, Tehran, Iran \\ ${ }^{3}$ School of Electrical Engineering, Shahid Rajaee Teacher Training University, Tehran, Iran \\ Correspondence should be addressed to Vahid Nayyeri; nayyeri@iust.ac.ir and Seyed Mohammad Hashemi; sm.hashemi@sru.ac.ir
}

Received 24 April 2019; Revised 25 January 2020; Accepted 6 March 2020; Published 7 April 2020

Academic Editor: Muhammad Ramlee Kamarudin

Copyright $\odot 2020$ Mohammad Reza Khawary et al. This is an open access article distributed under the Creative Commons Attribution License, which permits unrestricted use, distribution, and reproduction in any medium, provided the original work is properly cited.

This paper presents a novel ultracompact narrow bandpass filter with high selectivity. The proposed filter is composed of cascading two basic cells. Each cell is basically a microstrip line loaded with a quasiplanar resonator and series gaps which can be fabricated using a standard multilayer printed circuit board technology. The structure is analyzed through an equivalent circuit and full-wave simulations. The simulation results are compared with experimental measurements demonstrating a good agreement between them. The measurement indicates that the realized bandpass filter at the center frequency of $1 \mathrm{GHz}$ has a fractional bandwidth of 2.2\%. Most importantly, in comparison with other similar recent works, it is shown that the proposed filter has the smallest size.

\section{Introduction}

Design of very compact microwave devices compatible with the printed circuit board (PCB) and monolithic microwave integrated circuit fabrication technologies has gained great interest in the last decades. Electrically small planar resonators such as the split-ring resonators (SRRs) and their counterparts, i.e., complementary split-ring resonators (CSRRs), are key aspects that propose new design strategies to miniaturize planar microwave circuits [1]. In the case of the microstrip line, SRRs are patterned next to the strip line as shown in Figure 1(a), while the CSRRs, as shown in Figure 1(b), are etched on the ground plane. In general, for an arbitrary orientation of SRR or CSRR, it is necessary to account for both electric and magnetic coupling between the host line and the resonators [2]. However, when the ring slits are aligned in the orthogonal direction to the line axis (as in Figures 1(a) and 1(b)), the SRR and CSRR are coupled to the host line magnetically and electrically, i.e., they are derived by the magnetic field and the electric field parallel to the axis of the rings, respectively [2]. The authors in reference [][3] demonstrate that it is possible to design new resonators (such as broadside-coupled spiral resonators (BC-SRs)) with a higher level of miniaturization by combining two metal levels at both sides of a dielectric layer connected by vias with an appropriate topology (see Figure 1(c)). These miniaturized resonators can be effectively excited by the magnetic field produced by a microstrip line. For this purpose, it is imperative to open windows in the ground plane of the microstrip line in order to etch the lower side of the resonators. However, it should be noted that defected ground structures can lead to some electromagnetic compatibility (EMC) problems. Recently, Hashemi et al. [4] proposed to place a racemic mixture (with an equal amount of left- and right-handed inclusions) of BC-SRs between the signal strip and the ground plane (see Figure $1(\mathrm{~d})$ ). The resonators are oriented in a way that leads to their excitation by the electric field between the microstrip line and the ground plane. The advantages of this configuration are

(1) There is a strong coupling between the microstrip line and the resonators.

(2) The ground plane is preserved from being defected which prevents EMC problems. 


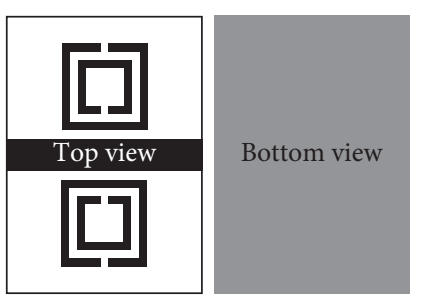

(a)

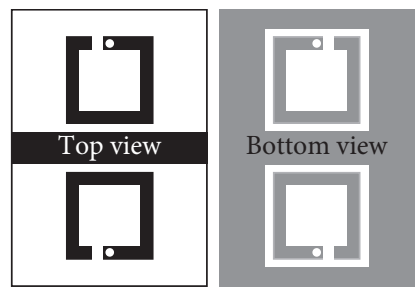

(c)

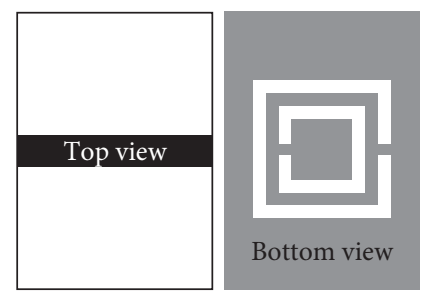

(b)

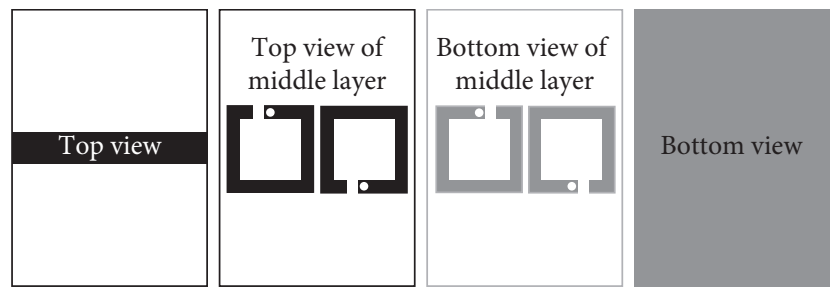

(d)

FIGURE 1: Different subwavelength planar resonators employed in the design of miniaturized planar microwave circuits: (a) SRRs, (b) CSRRs, (c) BC-SRs which are magnetically coupled to a line, and (d) BC-SRs which are electrically coupled to a line.

Electrically small planar resonators, including SRR and CSRR, have been used for the design of several types of filters, such as low-pass filters [5, 6] and band-pass filters (BPFs) [7-13]. Narrow BPFs with sharp selectivity and high rejection are increasingly demanded in modern microwave systems. To miniaturize such filters, SRRs [11-13], CSRRs [10], composite right-/ left-handed coplanar waveguide (CRLH-CPW) resonators [14], multisection stepped-impedance (SI) resonators [15], electromagnetic bandgap (EBG) resonators [16], and capacitively loaded coupled lines [17] have been applied. In this work, a new narrow BPF is investigated both theoretically and experimentally with a simple design and a higher level of miniaturization using BC-SRs which are electrically coupled to the line. The results of equivalent-circuit simulations, full-wave simulations, and experimental measurements are presented. It should be noted that a paper copied from this original work was previously published; however, it was recently retracted by the journal [18].

\section{Filter Topology}

Figure 2(a) shows the topology of a double-negative (DNG) cell conceptually proposed in [4], consisting of a microstrip line loaded with series gaps and a BC-SR which is electrically coupled to the line (the electrical coupling of BC-SRs to a microstrip line is also shown in Figure 1(d)). The equivalent circuit of the cell is depicted in Figure 2(b). The resonator's fundamental magnetic mode can be excited by both electric and magnetic fields applied parallel to the ring axis. However, it is demonstrated that excitation of magnetic modes of an electrically small particle by an external electric field is much stronger than the excitation of the same mode by an external magnetic field [19]. In the structure shown in Figure 2(a), the electric field produced by the microstrip line is along the axis of the BC-SR's ring, which effectively excites the fundamental magnetic mode of the BC-SR. Notice that the susceptibility to electric field is because of the strong electric field between the upper and lower rings that appear near the resonance [4]. Thus, this arrangement provides a strong electric coupling between the microstrip line and the BC-SR in a compact configuration.

In [4], it was demonstrated that this resonator provides negative permittivity in a narrow band after its resonance frequency $\left(f_{0}\right)$. On the contrary, the capacitive series gaps $\left(C_{g}\right)$ expose negative permeability before their plasma frequency $\left(f_{\mathrm{mp}}\right)$ [20]. Thus, as demonstrated in [4], this subwavelength structure with an appropriate topology supports backward-wave propagation in a narrow frequency band (see Figure 3). Therefore, the main idea behind this work is to utilize this DNG cell for design and fabrication of a compact narrow BPF.

Since the BC-SR is excited with the electrical field perpendicular to the substrate, for a better coupling, in this work, the transmission line above the particle is widened. This widening also prevents the gaps from becoming narrow to an extent that cannot be precisely fabricated (the fabrication technology limits the minimum gap size to $0.1 \mathrm{~mm}$ ). Furthermore, the line is tapered next to the widened section for a better matching.

As illustrated in Figure 2(a), this topology can be printed on the surfaces of a three-layer substrate. In this work, we used three layers of Rogers $4003 \mathrm{c}$ substrate with $\varepsilon_{r}=3.55$, $h=0.8 \mathrm{~mm}, \tan (\delta)=0.0027$, and $35 \mu \mathrm{m}$ copper metalization thickness. The BC-SR resonance frequency is determined by its dimensions. Among the dimension parameters indicated in Figure 2(a), the side length of the loop $(a)$ has the most influence in the resonance frequency. To realize the passband at $1 \mathrm{GHz}, a$ is set to $12.9 \mathrm{~mm}$. The series capacitive gaps lead to the structure behaving as a magnetic plasma, with negative valued permeability up to the plasma frequency $\left(f_{\mathrm{mp}}\right)$ which is basically the resonance frequency formed by the gap capacitance and the per-section inductance of the line. Hence, the structure must be designed so that the plasma frequency $\left(f_{\mathrm{mp}}\right)$ is higher than the resonance frequency $\left(f_{0}\right)$ of the BC-SRs (see Figure 3 ). Under this 


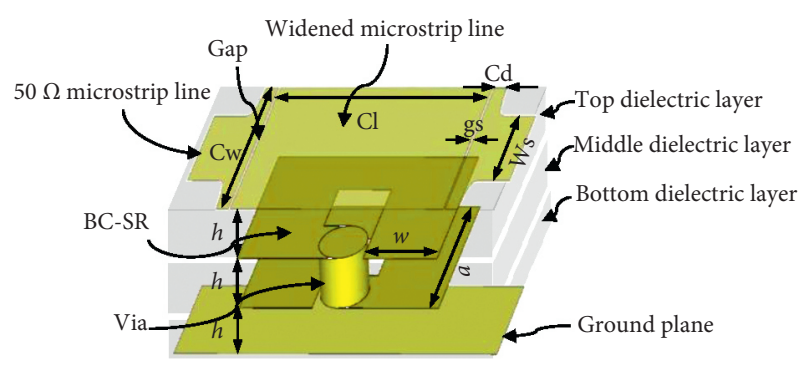

(a)

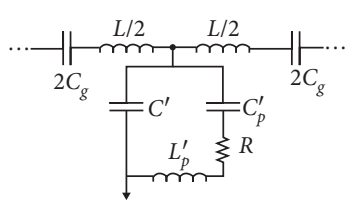

(b)

FIgURE 2: The DNG cell including a BC-SR electrically loaded to a microstrip line with series gaps: (a) a 3D layout and (b) the lumped element equivalent circuit.

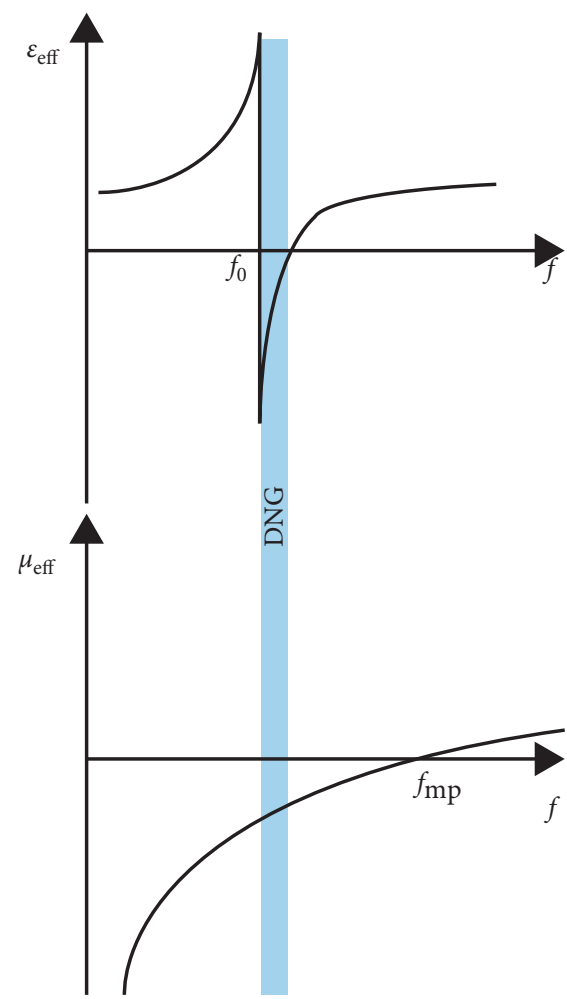

FIGURE 3: The $\varepsilon_{\text {eff }}$ and $\mu_{\text {eff }}$ models for the resonance particle and the series gap, respectively.

condition, there is a region where negative effective permeability and permittivity coexist. To meet this condition, our design gap size (gs) is set to $0.2 \mathrm{~mm}$. To extract the equivalent circuit parameters, shown in Figure 2(b), we applied the technique outlined in [4]. This technique is based on the comparison between the scattering parameters $\left(S_{11}\right.$ and $S_{21}$ ) which are obtained by a full-wave simulation and those obtained by the circuit analysis of the lumped-element equivalent model. The extracted values of the equivalent circuit elements are given in Table 1.

The results of full-wave electromagnetic and equivalent circuit simulations of the structure, shown in Figure 2, are depicted in Figure 4(a) which clearly shows a very good agreement between the results. A narrow passband at $1 \mathrm{GHz}$ with an insertion loss of around $1 \mathrm{~dB}$ and a return loss of
TABle 1: Extracted elements of the equivalent circuit.

\begin{tabular}{lccccc}
\hline$C_{p}^{\prime}$ & $L_{p}^{\prime}$ & $L$ & $C \prime$ & $C_{g}$ & $R$ \\
\hline $4.4 \mathrm{pF}$ & $7.3 \mathrm{nH}$ & $4.2 \mathrm{nH}$ & $10.2 \mathrm{pF}$ & $0.86 \mathrm{pF}$ & $0.6 \Omega$ \\
\hline
\end{tabular}

around $18 \mathrm{~dB}$ is observed in Figure 4(a). The electric field distribution over the cross section of the multilayer printed circuit board (at the middle of the structure) at the passband frequency (i.e., $1 \mathrm{GHz}$ ) is presented in Figure 4(b), showing that the electric field is along the axis of the BC-SR's ring.

The equivalent circuit model was also used to extract the effective relative permittivity $\left(\varepsilon_{\mathrm{r}}\right)$ and permeability $\left(\mu_{\mathrm{r}}\right)$ of the loaded transmission line using the method given in [21]. The results are shown in Figure 5. At the frequency of $1 \mathrm{GHz}$ (which is a passband frequency), the real parts of $\varepsilon_{\mathrm{r}}$ and $\mu_{\mathrm{r}}$ are negative, indicating a DNG transmission band. Moreover, considering the values of $\varepsilon_{\mathrm{r}}$ and $\mu_{\mathrm{r}}$ at $1 \mathrm{GHz}$, i.e., $\varepsilon_{\mathrm{r}} \approx-0.69$ and $\mu_{\mathrm{r}} \approx-0.02$ (notice that the imaginary parts of $\varepsilon_{\mathrm{r}}$ and $\mu_{\mathrm{r}}$ are negligible), the impedance ratio of the loaded line is found as

$$
Z=\sqrt{\frac{\mu_{\mathrm{r}} \mu_{0}}{\varepsilon_{\mathrm{r}} \varepsilon_{0}}} \approx \sqrt{\frac{-0.02}{-0.69}} \times 377 \approx 64 \Omega,
$$

which is fairly close to $50 \Omega$ and demonstrates a passband around $1 \mathrm{GHz}$.

To achieve a deeper rejection and also a sharper passband, as shown in the inset of Figure 6(a), two DNG cells are cascaded, where cell I and cell II contain a left-handed and a right-handed chiral resonator, respectively. These two cells are identical in shape and dimensions, and just their handness are opposite (see Figure 1(d) showing two identical resonators with opposite handness) to remove the chirality of the medium [4]. The results of the full-wave electromagnetic simulation (obtained using HFSS) and the equivalent circuit analysis are depicted in Figures 6(a) and 6(b) which are in a good agreement confirming the validity of the equivalent circuit model. A narrow passband around $1 \mathrm{GHz}$ is obvious in the figure.

\section{Experimental Results}

As mentioned earlier, three layers of Rogers RO4003c (with thickness of $0.8 \mathrm{~mm}$ ) were used to fabricate the proposed 


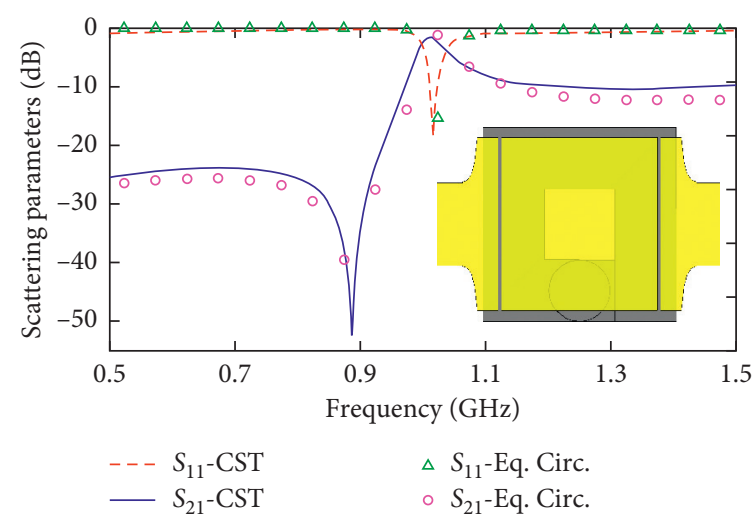

(a)

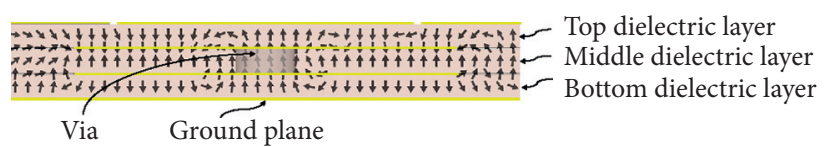

(b)

Figure 4: (a) The scattering parameters of the DNG cell, and (b) the electric field distribution over the cross section of the multilayer printed circuit board (at the middle of the structure) at $1 \mathrm{GHz}$, where the parameters indicated in Figure $1(\mathrm{a})$ are set as, $a=12.9 \mathrm{~mm}, w=4.3 \mathrm{~mm}$, $\mathrm{Ws}=5.5 \mathrm{~mm}, \mathrm{Cl}=10 \mathrm{~mm}, \mathrm{Cw}=11.4 \mathrm{~mm}, \mathrm{Cd}=1.4 \mathrm{~mm}, \mathrm{gs}=0.2 \mathrm{~mm}$, and $h=0.8 \mathrm{~mm}$.

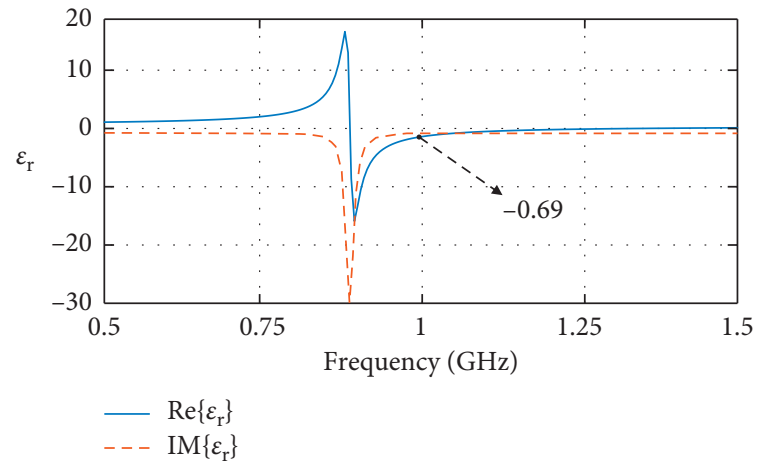

(a)

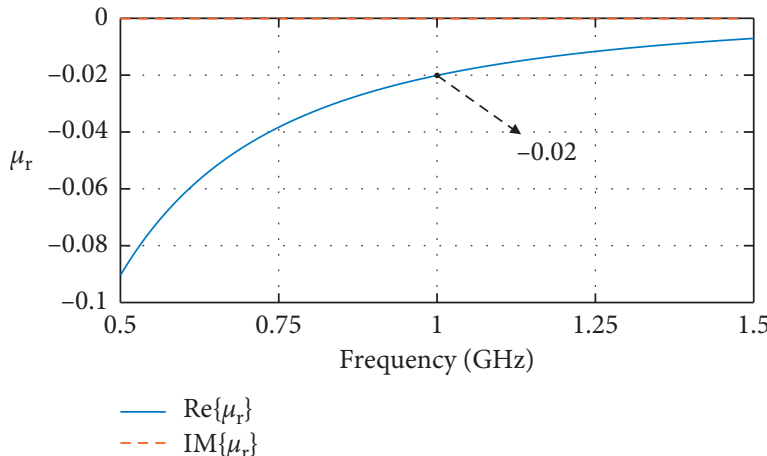

(b)

FIgURE 5: The effective relative permittivity (a) and permeability (b) of the loaded transmission line.

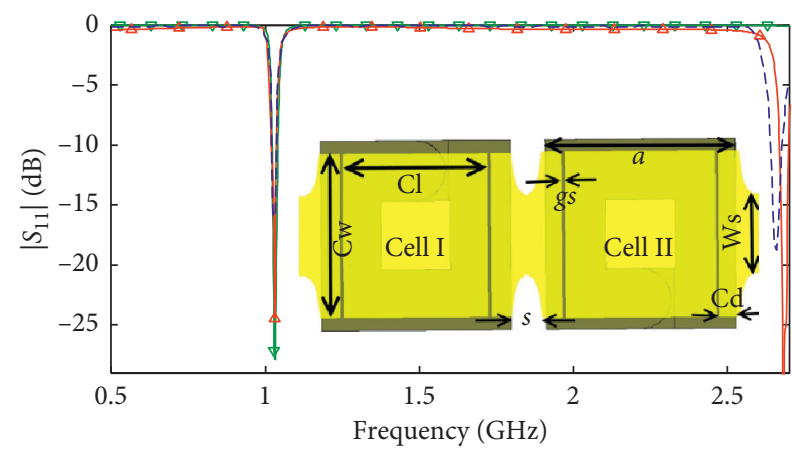

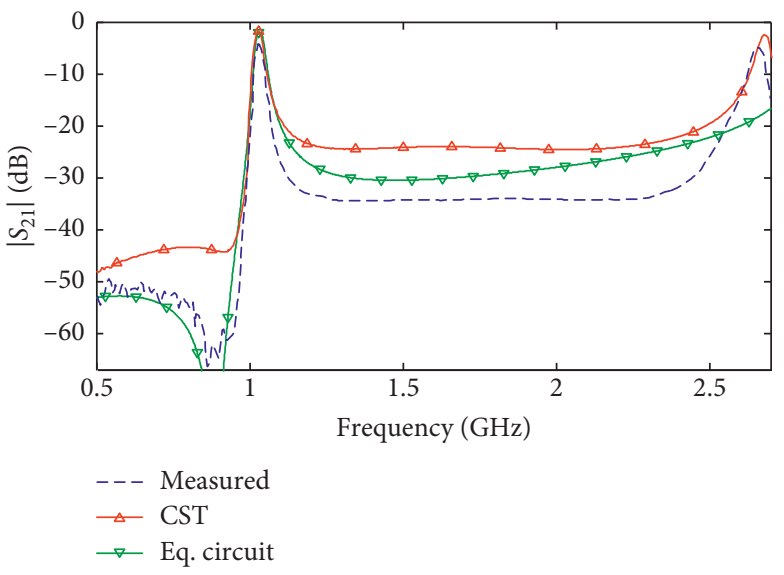

(b)

Figure 6: (a) $\left|S_{11}\right|$ and (b) $\left|S_{21}\right|$ of two cascaded DNG cells (where $a=12.9 \mathrm{~mm}, w=4.3 \mathrm{~mm}$, Ws $=5.5 \mathrm{~mm}, \mathrm{Cl}=10 \mathrm{~mm}, \mathrm{Cw}=11.4 \mathrm{~mm}$, $\mathrm{Cd}=1.4 \mathrm{~mm}$, gs $=0.2 \mathrm{~mm}$, and $s=2.1 \mathrm{~mm}$ ).

filter. As shown in Figure 7(a), the transmission line with gaps was printed on the top surface of the top layer. Two metal rings were printed on each sides of the middle layer (can be seen in Figures 7(b) and 7(c)) and were connected to each other using copper ribbons to form quasiplanar resonators. The bottom layer was the ground plane. Finally, 


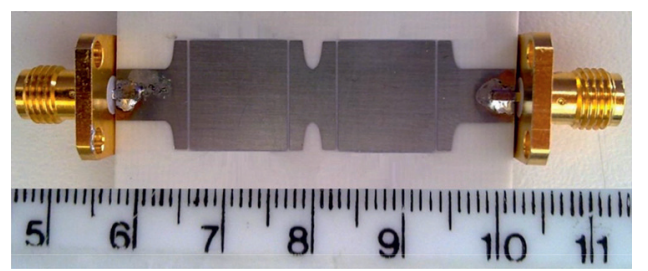

(a)

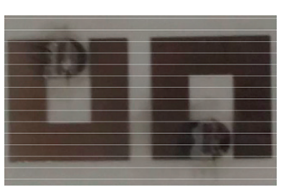

(b)

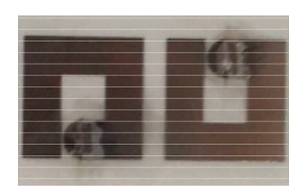

(c)

Figure 7: The layers of the fabricated filter: (a) top layer, (b) top view of the middle layer, and (c) bottom view of the middle layer (notice that the bottom layer (not shown in this figure) is the ground plane).

TABLE 2: Comparison between the proposed filter and some other compact narrow BPFs reported in the literature (sorted by their size in the descending order).

\begin{tabular}{|c|c|c|c|c|c|c|c|}
\hline Reference & Resonator & $f_{0}(\mathrm{GHz})$ & FBW (\%) & Size $\left[\lambda_{0} \times \lambda_{0}\right]$ & $\mathrm{IL}(\mathrm{dB})$ & No. of metal layers & $\varepsilon_{\mathrm{r}}$ of the substrates \\
\hline [11] & SRR & 4.6 & 1.7 & $0.46 \times 0.23$ & 5.2 & 2 & 2.2 \\
\hline [22] & CSRR & 4.9 & 4 & $0.41 \times 0.16$ & 2.8 & 2 & 2.2 \\
\hline [13] & SRR & 0.9 & 2.9 & $0.25 \times 0.07$ & 5.1 & 3 & 10.2 \\
\hline [16] & EBG & 5 & 3.7 & $0.23 \times 0.08$ & 2.8 & 2 & 10.2 \\
\hline [14] & CRLH-CPW & 5 & 6 & $0.2 \times 0.08$ & 2.5 & 2 & 10.2 \\
\hline [23] & Loaded stub & 2.4 & 3.5 & $0.2 \times 0.17$ & 2.3 & 2 & 3.5 \\
\hline$[24]$ & SI-CSRR & 8.4 & 7.9 & $0.2 \times 0.1$ & 1.5 & 2 & 2.2 \\
\hline [25] & Square SRR & 2.4 & 12.1 & $0.18 \times 0.07$ & 0.3 & 2 & 10.2 \\
\hline [12] & S-shaped SRR & 1.25 & 12 & $0.18 \times 0.04$ & 2.4 & 2 & 10.2 \\
\hline [15] & SI resonator & 2.4 & 10.8 & $0.16 \times 0.16$ & 5.8 & 2 & 4.5 \\
\hline [10] & CSRR & 1 & 8 & $0.16 \times 0.07$ & 1.5 & 2 & 10.2 \\
\hline This work & BC-SR & 1 & 2.2 & $0.11 \times 0.04$ & 4.2 & 4 & 3.5 \\
\hline
\end{tabular}

three layers were stuck together using glue. The results obtained from the equivalent circuit analysis, the full-wave simulation, and the measurement are shown in Figures 6(a) and 6(b), demonstrating a significant agreement between them; however, using copper ribbons instead of vias has an effect on the resonance frequency of the resonators, which appears by a slight frequency shift in the measurement results. In addition, in Figure 6(b), the measured $S_{21}$ shows an insertion loss of $4.2 \mathrm{~dB}$ at the central frequency; however, the simulated insertion loss is around $1.8 \mathrm{~dB}$. This extra loss is due to the use of glue for bonding the boards. Using a lowloss prepreg (for example, Rogers RO4450 Bondply) instead of glue can reduce the insertion loss considerably.

Finally, the proposed filter is compared with some other compact narrow BPFs reported in the literature in terms of resonator type, center frequency $\left(f_{0}\right)$, fractional bandwidth (FBW), length, insertion loss (IL), number of metal layers in the design, and dielectric constant of the substrates. The comparison is shown in Table 2 which is sorted by filter size in the descending order. In this table, it can be seen that while in our work a high dielectric constant is not used, with a size of $0.11 \lambda_{0} \times 0.03 \lambda_{0}$, the proposed filter is the most compact. It is worth mentioning that the application of a high dielectric constant substrate (for example, with $\varepsilon_{\mathrm{r}}=10.2$ as in the most referenced works in Table 2) further compacts the design because the guided wavelength in such a medium (with a high dielectric constant) is much shorter. At the same time, with an FBW $=2.2 \%$, the proposed filter is the second most selective (narrowest). On the contrary, the proposed design includes four metal layers which are patterned on three dielectric layers and so a multilayer PCB fabrication procedure is needed; however, the other works (except [13]) include only two metal layers and are more easily fabricated by pattering on the both sides of a single-layer PCB. In Table 2, it should be also noted that with an insertion loss of $4.2 \mathrm{~dB}$, the fabricated structure is considered as a high-loss filter; however, the insertion loss can be considerably reduced by applying a low-loss prepreg instead of glue for bonding the layers.

\section{Conclusion}

A narrow bandpass filter with simple design, backwardpropagation passband, compact size, and high selectivity based on the quasiplanar resonator was presented. The results of the equivalent circuit analysis, the full-wave simulation, and the measurement were presented. In comparison with other similar works, it was shown that the proposed filter is the most compact and is yet a very narrow band.

\section{Data Availability}

No data were used to support this study.

\section{Conflicts of Interest}

The authors declare that they have no conflicts of interest. 


\section{References}

[1] R. Marques, F. Martin, and M. Sorolla, Metamaterials with Negative Parameters: Theory, Design and Microwave Applications, Wiley, New York, NY, USA, 2008.

[2] J. Naqui, M. Duran-Sindreu, and F. Martin, "Modeling splitring resonator (SRR) and complementary split-ring resonator (CSRR) loaded transmission lines exhibiting cross-polarization effects," IEEE Antennas and Wireless Propagation Letters, vol. 12, pp. 178-181, 2013.

[3] F. Aznar, J. García-García, M. Gil, J. Bonache, and F. Martín, "Strategies for the miniaturization of metamaterial resonators," Microwave and Optical Technology Letters, vol. 50, no. 5, pp. 1263-1270, 2008.

[4] S. M. Hashemi, M. Soleimani, and S. A. Tretyakov, "Compact negative-epsilon stop-band structures based on double-layer chiral inclusions," IET Microwaves, Antennas \& Propagation, vol. 7, no. 8, pp. 621-629, 2013.

[5] B. Wu, B. Li, and C. Liang, "Design of lowpass filter using a novel split-ring resonator defected ground structure," $\mathrm{Mi}$ crowave and Optical Technology Letters, vol. 49, no. 2, pp. 288-291, 2007.

[6] X. Chen, L. Weng, and X. Shi, "Novel complementary split ring resonantor DGS for low-pass filter design," Microwave and Optical Technology Letters, vol. 51, no. 7, pp. 1748-1751, 2009.

[7] J. Garcia-Garcia, J. Bonache, I. Gil, F. Martin, R. Marques, and F. Falcone, "Comparison of electromagnetic band gap and split-ring resonator microstrip lines as stop band structures," Microwave and Optical Technology Letters, vol. 44, no. 4, pp. 376-379, 2005.

[8] J. Bonache, F. Martín, I. Gil, J. García-García, R. Marqués, and M. Sorolla, "Microstrip bandpass filters with wide bandwidth and compact dimensions," Microwave and Optical Technology Letters, vol. 46, no. 4, pp. 343-346, 2005.

[9] P. Mondal, M. K. Mandal, A. Chaktabarty, and S. Sanyal, "Compact bandpass filters with wide controllable fractional bandwidth," IEEE Microwave and Wireless Components Letters, vol. 16, no. 10, pp. 540-542, 2006.

[10] J. Bonache, I. Gil, J. Garcia-Garcia, and F. Martin, "Novel microstrip bandpass filters based on complementary split-ring resonators," IEEE Transactions on Microwave Theory and Techniques, vol. 54, no. 1, pp. 265-271, 2006.

[11] A. L. Borja, J. Carbonell, V. E. Boria, J. Casc 'on, and D. Lippens, "A $2 \%$ bandwidth C-band filter using cascaded split ring resonators," IEEE Antennas and Wireless Propagation Letters, vol. 9, pp. 256-159, 2010.

[12] A. K. Horestani, M. Duran-Sindreu, J. Naqui, C. Fumeaux, and F. Martin, "Coplanar waveguides loaded with S-shaped split-ring resonators: modeling and application to compact microwave filters," IEEE Antennas and Wireless Propagation Letters, vol. 13, pp. 1349-1352, 2014.

[13] A. Genc, R. Baktur, and R. J. Jost, "Dual-bandpass filters with individually controllable passbands," IEEE Transactions on Components, Packaging and Manufacturing Technology, vol. 3, no. 1, pp. 105-112, 2013.

[14] S.-G. Mao, M.-S. Wu, Y.-Z. Chueh, and C. H. Chen, "Modeling of symmetric composite right/left-handed coplanar waveguides with applications to compact bandpass filters," IEEE Transactions on Microwave Theory and Techniques, vol. 53, no. 11, pp. 3460-3466, 2005.

[15] H. Zhang and K. J. Chen, "Miniaturized coplanar waveguide bandpass filters using multisection stepped-impedance resonators," IEEE Transactions on Microwave Theory and Techniques, vol. 54, no. 2, pp. 1090-1095, 2006.
[16] S.-G. Mao and Y.-Z. Chueh, "Coplanar waveguide bandpass filters with compact size and wide spurious-free stopband using electromagnetic bandgap resonators," IEEE Microwave and Wireless Components Letters, vol. 17, no. 3, pp. 181-183, 2007.

[17] M. Orellana, J. Selga, P. Velez et al., "Design of capacitively loaded coupled-line bandpass filters with compact size and spurious suppression," IEEE Transactions on Microwave Theory and Techniques, vol. 65, no. 4, pp. 1235-1248, 2017.

[18] Retracted: design of a narrow bandwidth bandpass filter using compact spiral resonator with chirality," Active and Passive Electronic Components, vol. 2019, Article ID 7262158, , 2019.

[19] A. Serdiukov, I. Semchenko, S. Tertyakov, and A. Sihvola, Electromagnetics of BiAnisotropic Materials: Theory and Applications, Gordon and Breach, New York, NY, USA, 2001.

[20] G. V. Eleftheriades, O. Siddiqui, and A. K. Iyer, "Transmission line models for negative refractive index media and associated implementations without excess resonators," IEEE Microwave and Wireless Components Letters, vol. 13, no. 2, pp. 51-53, 2003.

[21] C. Caloz and T. Itoh, Electromagnetic Metamaterials: Transmission Line Theory and Microwave Applications, John Wiley \& Sons, Hoboken, NJ, USA, 2005.

[22] B. Yin, Z. Lin, X. Cai, H. Hao, W. Luo, and W. Huang, "A novel compact CRLH bandpass filter on CSRR-loaded substrate integrated waveguide cavity," Progress in Electromagnetics Research M, vol. 75, pp. 121-129, 2018.

[23] M.-C. Tang, T. Shi, and X. Tan, "A novel triple-mode hexagon bandpass filter with meander line and central-loaded stub," Microwave and Optical Technology Letters, vol. 58, no. 1, pp. 9-12, 2016.

[24] Y. M. Huang, G. Wang, W. Jiang, T. Huang, and Z. Shao, "Half-mode substrate integrated waveguide bandpass filter loaded with horizontal-asymmetrical stepped-impedance complementary split-ring resonators," Electronics Letters, vol. 52, no. 12, pp. 1034-1036, 2016.

[25] A. Belmajdoub, "Design, optimization and realization of compact bandpass filter using two identical square open-loop resonators for wireless communications systems," Journal of Instrumentation, vol. 14, p. 09012, 2019. 